\title{
BOUNDEDNESS VERSUS PERIODICITY OVER COMMUTATIVE LOCAL RINGS
}

\author{
VESSELIN N. GASHAROV AND IRENA V. PEEVA
}

\begin{abstract}
Over commutative graded local artinian rings, examples are constructed of periodic modules of arbitrary minimal period and modules with bounded Betti numbers, which are not eventually periodic. They provide counterexamples to a conjecture of D. Eisenbud, that every module with bounded Betti numbers over a commutative local ring is eventually periodic of period 2 . It is proved however, that the conjecture holds over rings of small length.
\end{abstract}

\section{INTRODUCTION}

This paper considers the relations between the structure of finitely generated modules over local noetherian rings and the asymptotic behavior of their sequences of Betti numbers, along the lines suggested by the work of Eisenbud [5] and of Avramov [2]. Particular attention is given to modules whose Betti numbers are bounded. In order to make precise statements, we introduce some notation that will be in force for the rest of the paper.

Let $R$ be a commutative noetherian local ring with maximal ideal $m$ and residue field $k=R / m$. As usual, we denote by edim $R$ the embedding dimension of $R$, that is, the minimal number of generators of $m$, and by depth $R$ the longest length of a regular sequence contained in $m$. If $M$ is a finitely generated $R$-module we denote by $\nu(M)$ its minimal number of generators. Let

$$
\text { F: } F_{0} \stackrel{d_{1}}{\longleftarrow} F_{1} \stackrel{d_{2}}{\longleftarrow} F_{2} \longleftarrow \cdots
$$

be a minimal free resolution of $M$ over $R$. The $n$th Betti number of $M$ over $R$ is $b_{n}=b_{n}^{R}(M)=\operatorname{rank} F_{n}$, and $\operatorname{Syz}_{n}^{R}(M)=\operatorname{Coker}\left(d_{n+1}: F_{n+1} \rightarrow F_{n}\right)$ is called the $n$th syzygy of $M$ over $R$. Following $[1,2]$ we say $M$ has complexity $c$, and write $\mathrm{cx}_{R} M=c$, if $c \geq 0$ is the least integer for which there exists a real number $A>0$ such that $b_{n}^{R}(M) \leq A n^{c-1}$ holds for $n \geq 1$; if no such $c$ exists, set $\mathrm{cx}_{R} M=\infty$.

From a homological point of view, the simplest modules are those with $b_{n}^{R}(M)=0$ for sufficiently large $n$; thus the projective dimension, $\operatorname{pd}_{R} M$,

Received by the editors March 21, 1988 and, in revised form, October 6, 1988.

1980 Mathematics Subject Classification (1985 Revision). Primary 13D25, 13H10; Secondary 18G10, $20 \mathrm{C} 99$.

Key words and phrases. Betti numbers, periodic module, minimal free resolution. 
is finite exactly when $\mathrm{cx}_{R} M=0$. The next natural question is what does $\mathrm{cx}_{R} M \leq 1$ mean? A simple reason for this to happen is the presence of periodicity. A module $M$ is said to be eventually periodic of period $q>0$ if there is a nonnegative integer $s$ such that $\operatorname{Syz}_{s}^{R}(M) \cong \operatorname{Syz}_{s+q}^{R}(M)$. If $s=0, M$ is called periodic of period $q$. Modules of complexity one seem to occur rarely, and in [5] Eisenbud stated the following:

Conjecture. If $M$ is an $R$-module with bounded Betti numbers, then $M$ is eventually periodic; a periodic $R$-module has period 2 .

The main cases in which the conjecture has been proved are summarized as follows:

(1.0) Theorem. Let $M$ be a finitely generated $R$-module such that the sequence $\left\{b_{n}^{R}(M)\right\}_{n \geq 0}$ is bounded. Then $M$ is eventually periodic of period 2 when one of the following assumptions holds:

(i) (Eisenbud [5, Theorem 4.1]) $R$ is a complete intersection;

(ii) (Avramov [2, Theorem 1.6]), $\operatorname{edim} R-\operatorname{depth} R \leq 3$, or $\operatorname{edim} R-$ depth $R=4$ and $R$ is Gorenstein.

In $\S 2$ a proof of the following result is given:

(1.1) Theorem. Let $R$ be a Cohen-Macaulay local ring of multiplicity $\leq 7$ and $\operatorname{edim} R-\operatorname{depth} R \geq 4$, or a Gorenstein local ring of multiplicity $\leq 11$ and $\operatorname{edim} R-\operatorname{depth} R \geq 5$. Then for every finitely generated $R$-module $M$ either $\operatorname{pd}_{R} M<\infty$ or the sequence $\left\{b_{n}^{R}(M)\right\}_{n \geq \nu(M)+\operatorname{depth} R+1}$ is strictly increasing.

An immediate consequence of (1.0) and (1.1) is the validity of Eisenbud's conjecture over rings of small multiplicity:

(1.2) Theorem. Let $R$ be a Cohen-Macaulay local ring of multiplicity $\leq 7$ or a Gorenstein local ring of multiplicity $\leq 11$. If $M$ is a finitely generated $R$ module with bounded Betti numbers, then $M$ is eventually periodic of period 2 .

The main results of this paper are the constructions of counterexamples to Eisenbud's conjecture. They establish the following:

(1.3) Theorem. There are local graded artinian rings of embedding dimension 4 and length 8, and local graded Gorenstein rings of embedding dimension 5 and length 12 , over which both claims of the conjecture fail.

The examples are given in $\S 3$, where their "minimality" is also discussed.

Thus the problem "What is the reason for the Betti numbers of $M$ to be bounded?" remains open. In particular, it is not known whether the boundedness of the Betti numbers implies that they become constant for large $n$, a question asked by Ramras. 
The final $\S 4$ contains some remarks on the restrictions on $k$ imposed by our constructions. In particular, it is shown that the first statement of Eisenbud's conjecture holds when $R$ is artinian with $k$ algebraic over a finite field, but the second statement fails with any $k$.

We are grateful to Luchezar Avramov for his guidance during this research and for help in writing the paper.

\section{MODUleS OVER ARTINIAN RINGS}

For a finitely generated $R$-module $M, l(M)$ denotes its length. Throughout this section, we use the following notation:

$$
\begin{aligned}
& e_{i}=\operatorname{dim}_{k} m^{i} / m^{i+1} \quad(i>0), \\
& \operatorname{Hilb}_{R}(t)=1+\sum_{i=1}^{\infty} e_{i} t^{i}, \\
& e=\nu(m)=\operatorname{edim} R=e_{1}, \\
& h=\min \left\{i \mid m^{i+1}=0\right\}, \\
& l=l(R) .
\end{aligned}
$$

Over an artinian ring $R$, there is an obvious relation between the number of generators of a submodule $M$ of $m^{n} R^{b}$ and the length of $m^{n}$, namely: $\nu(M) \leq l(M) \leq l\left(m^{n}\right) b$. Our first lemma shows that this can be improved in a useful way.

(2.1) Lemma. Let $R$ be an artinian ring and let $M$ be a finitely generated $R$-module, such that $M \subseteq m^{n} R^{b}, b \geq 1, n \geq 0$. Then

$$
\nu(M) \leq\left(l\left(m^{n}\right)+n-h\right) b .
$$

Proof. By descending induction on $n$. When $n=h$, the claim coincides with the remark made above. Now let $n<h$ and suppose that the assertion has already been proved for $n+1$. Let $v_{1}, \ldots, v_{s}$ be a generating set of $M$. We may assume that $v_{1}, \ldots, v_{p}(p \leq s)$ are contained in a minimal generating set of $m^{n} R^{b}$ and $v_{p+1}, \ldots, v_{s}$ are in $m^{n+1} R^{b}$.

Consider first the case when $p \leq\left(e_{n}-1\right) b$. Writing $M^{\prime}$ for $\left(v_{p+1}, \ldots, v_{s}\right) R$, we have that $\nu(M) \leq p+\nu\left(M^{\prime}\right)$. Using the induction hypothesis for $M^{\prime} \subseteq$ $m^{n+1} R^{b}$ we obtain

$$
\begin{aligned}
\nu(M) & \leq p+\nu\left(M^{\prime}\right) \leq\left(e_{n}-1\right) b+\left(l\left(m^{n+1}\right)+n+1-h\right) b \\
& =\left(l\left(m^{n}\right)+n-h\right) b .
\end{aligned}
$$

Suppose now $\left(e_{n}-1\right) b<p$, set $q=e_{n} b-p$, and note that $0 \leq q<b$. Let $v_{1}, \ldots, v_{p}, w_{1}, \ldots, w_{q}$ be a minimal generating set for $m^{n} R^{b}$. We may take the $w_{i}$ 's of the form $\left(0, \ldots, 0, y_{i}, 0, \ldots, 0\right)$ with the nonzero element $y_{i} \in m^{n}$ in $r_{i}$ th place. Furthermore, permuting the summands of $R^{b}$ if necessary, we assume that $r_{i}>b-q$ for $1 \leq i \leq q$, i.e., $w_{i} \in m^{n} R^{q}$, where the decomposition $R^{b}=R^{b-q} \oplus R^{q}$ is taken with respect to the standard basis. Thus for $p+1 \leq j \leq s$ we have $v_{j}=\sum_{i=1}^{p} z_{i j} v_{i}+v_{j}^{\prime}$ with $z_{i j} \in m$ and 
$v_{j}^{\prime} \in m^{n+1} R^{q}$. In particular, $M=\left(v_{1}, \ldots, v_{p}, v_{p+1}^{\prime}, \ldots, v_{s}^{\prime}\right) R$. Applying the induction hypothesis to $M^{\prime \prime}=\left(v_{p+1}^{\prime}, \ldots, v_{s}^{\prime}\right) R \subseteq m^{n+1} R^{q}$ we obtain

$$
\begin{aligned}
\nu(M) & \leq p+\nu\left(M^{\prime \prime}\right) \leq e_{n} b-q+\left(l\left(m^{n+1}\right)+n+1-h\right) q \\
& \leq e_{n} b+\left(l\left(m^{n+1}\right)+n-h\right) b=\left(l\left(m^{n}\right)+n-h\right) b .
\end{aligned}
$$

(2.2) Proposition. Let $R$ be an artinian ring and let $M$ be a finitely generated $R$-module. Then $b_{n+1}^{R}(M) \geq(2 e-l+h-1) b_{n}^{R}(M)$ for $n \geq \nu(M)$.

Proof. We may suppose $M$ is not free and $2 e-l+h-1 \geq 1$ (otherwise the assertion is trivial). There is then an $n \leq \nu(M)$ such that $b_{n} \geq b_{n-1}=b$. In order to establish the required inequality, we construct a sufficiently large set of elements in $\operatorname{Ker} d_{n}$ and show that it can be extended to a minimal system of generators of $\operatorname{Ker} d_{n}$.

Let $x_{1}, \ldots, x_{e}$ be a minimal generating set of $m$. Consider the elements

$$
\left\{w_{s} \mid 1 \leq s \leq e b_{n}\right\}=\left\{\left(0, \ldots, 0, x_{i}, 0, \ldots, 0\right) \in R^{b_{n}}\right.
$$

$$
\text { where } \left.x_{i} \text { is in the } j \text { th place } \mid 1 \leq i \leq e, 1 \leq j \leq b_{n}\right\} \text {. }
$$

Since $d_{n}\left(w_{s}\right) \in m^{2} R^{b}$ we know by (2.1) that $q \leq\left(l\left(m^{2}\right)+2-h\right) b$ among them generate $\left(d_{n}\left(w_{1}\right), \ldots, d_{n}\left(w_{e b_{n}}\right)\right) R$. Renumbering, if necessary, we have that $d_{n}\left(w_{i}\right)=\sum_{j=1}^{q} y_{i j} d_{n}\left(w_{j}\right), q<i \leq e b_{n}, y_{i j} \in R$. Hence $u_{i}=w_{i}-$ $\sum_{j=1}^{q} y_{i j} w_{j}$ are in $\operatorname{Ker} d_{n}$. Note that the images of $u_{i}$ are linearly independent in $m R^{b_{n}} / m^{2} R^{b_{n}}$, hence in $\operatorname{Ker} d_{n} / m \operatorname{Ker} d_{n}$. Thus the $u_{i}$ 's are contained in a minimal generating set of $\operatorname{Ker} d_{n}$. Their number, $e b_{n}-q$, does not exceed $b_{n+1}$, hence

$$
\begin{aligned}
b_{n+1} & \geq e b_{n}-q \geq e b_{n}-\left(l\left(m^{2}\right)+2-h\right) b_{n-1} \geq e b_{n}-\left(l\left(m^{2}\right)+2-h\right) b_{n} \\
& =(2 e-l+h-1) b_{n}
\end{aligned}
$$

In particular, $b_{n+1} \geq b_{n}$ and the argument can be iterated.

(2.3) Corollary. Let $R$ and $M$ be as in (2.2).

(i) If $2 e-l+h-1=1$, then there exists a $t$ with $\nu(M)-1 \leq t \leq \infty$ such that $b_{n}^{R}(M)=b_{n+1}^{R}(M)$ for $\max (\nu(M)-1,1) \leq n<t$ and $b_{n}^{R}(M)<b_{n+1}^{R}(M)$ for $n \geq t$.

(ii) If $2 e-l+h-1 \geq 2$ then the sequence $\left\{b_{n}^{R}(M)\right\}_{n \geq \nu(M)}$ is strictly increasing and has strong exponential growth; i.e., there exist real numbers $B \geq A>1$ such that $A^{n} \leq b_{n}^{R}(M) \leq B^{n}$ holds for all sufficiently large $n$.

Proof. (i) It only remains to remark that under the hypothesis of (i), if $b_{n}>$ $b_{n-1}$, then the third inequality in $(*)$ is strict, hence $b_{n+1}>b_{n}$.

(ii) The upper bound is well known to hold for arbitrary modules; e.g., see $[2,(2.5)]$. For the lower bound apply (2.2).

(2.4) Corollary. Eisenbud's conjecture holds over artinian rings with $m^{2}=0$ and over Gorenstein rings with $\mathrm{m}^{3}=0$.

Proof. If edim $R \leq 3$, apply (1.0.ii). If $\operatorname{edim} R>3$, apply (2.3.ii). 
It should be noted that (2.4) easily follows from the results of Eisenbud [5, Theorem 4.1] and of Lescot [7, Theorem B and Proposition 3.9].

(2.5) Lemma. Let $R$ be an artinian Gorenstein ring with $l\left(m^{h-1}\right) \geq 3$. If $M$ is an $R$-submodule of the $R$-module $m^{h-1} R^{b}, b \geq 1$ and $\nu(M)=\left(l\left(m^{h-1}\right)-1\right) b$, then $M=m^{h-1} R^{b}$.

Proof. Choose elements $v_{1}, \ldots, v_{p} \in \operatorname{soc} M$, whose images form a $k$-basis of the image of $\operatorname{soc} M \hookrightarrow M \rightarrow M / m M$. Complete $v_{1}, \ldots, v_{p}$ to a minimal generating set of $M$, say $v_{1}, \ldots, v_{s}$. Set $M^{\prime}=\left(v_{1}, \ldots, v_{p}\right) R \subseteq \operatorname{soc} M, M^{\prime \prime}=$ $\left(v_{p+1}, \ldots, v_{s}\right) R$. Obviously, $m M^{\prime \prime}=m M \subseteq \operatorname{soc} R^{b}$ and $M^{\prime} \cap m M=0$, hence there is a $k$-vector space $N$ with basis $n_{1}, \ldots, n_{c}$ such that $\operatorname{soc} R^{b} \supseteq N \supseteq M^{\prime}$ and $N \oplus m M=\operatorname{soc} R^{b}$. Since $R$ is a zero-dimensional Gorenstein ring, $R^{b}$ is an injective $R$-module. Therefore $R^{b}=P \oplus Q$ for some $R$-module $Q$, where $P \cong R^{c}$ is the injective envelope of $N$.

We are going to prove that $P \cap M^{\prime \prime}=0$. Since $P$ is an essential extension of $N$, it suffices to show that $N \cap M^{\prime \prime}=0$. This is obvious from the relations $N \cap M^{\prime \prime} \subseteq \operatorname{soc} R^{b} \cap M=\operatorname{soc} M=M^{\prime} \oplus m M^{\prime \prime}$. Thus, the canonical map $R^{b} \rightarrow R^{b} / P \cong R^{b-c}$ is injective on $M^{\prime \prime}$. Since the image of $M^{\prime \prime}$ is contained in $m^{h-1} R^{b-c}$, it follows by Lemma $(2.1)$ that $\nu\left(M^{\prime \prime}\right) \leq e_{h-1}(b-c)$. This implies $e_{h-1} b=\nu(M) \leq \nu\left(M^{\prime}\right)+\nu\left(M^{\prime \prime}\right) \leq \nu(N)+\nu\left(M^{\prime \prime}\right) \leq c+e_{h-1}(b-c)$, hence $c=0$, i.e., $N=0$. We have shown that $m M=\operatorname{soc} R^{b}=m^{h} R^{b}$, so that $M / m M \hookrightarrow$ $m^{h-1} R^{b} / m^{h} R^{b}$. But $\operatorname{dim}_{k} M / m M=\nu(M)=e_{h-1} b=\operatorname{dim}_{k} m^{h-1} R^{b} / m^{h} R^{b}$; therefore $M / m M=m^{h-1} R^{b} / m^{h} R^{b}$, which yields $M=m^{h-1} R^{b}$.

(2.6) Proposition. Let $R$ be a Gorenstein ring with $h=3$. Then $l \leq 2(e+1)$. If $l \neq 2(e+1)$, then either $M$ is free or the sequence $\left\{b_{n}^{R}(M)\right\}_{n \geq \nu(M)+1}$ is strictly increasing.

Proof. Since $R$ is self-injective, one has

$$
l\left(0: m^{2}\right)=l(R)-l\left(m^{2}\right)=1+e_{1}+e_{2}+1-\left(e_{2}+1\right)=e_{1}+1 .
$$

Now $m^{4}=0$ gives the inclusion $m^{2} \subseteq\left(0: m^{2}\right)$; hence $1+e_{2} \leq 1+e_{1}$, i.e., $e_{2} \leq e_{1}$.

We have shown $l \leq 2(e+1)$. if $l<2 e+1$, our claim follows from (2.3.ii). Hence we assume $l=2 e+1$, i.e., $\operatorname{Hilb}_{R}(t)=1+e t+(e-1) t^{2}+t^{3}$. By (2.3.i) it suffices to show that $b_{n}<b_{n+1}$ for some $n \leq \nu(M)+1$. Suppose the contrary. By the same corollary we then have $b=b_{i-1}=b_{i}=b_{i+1}=b_{i+2} \leq b_{i+3}$ for $i=\nu(M)$. Set $I_{n-1}=d_{n}\left(m R^{b_{n}}\right)$. The exact sequences

$$
R^{b_{n+1}} \stackrel{d_{n+1}}{\longrightarrow} m R^{b_{n}} \stackrel{d_{n}}{\longrightarrow} I_{n-1} \longrightarrow 0
$$

yield for $n=i, i+1, i+2$ :

$$
\nu\left(I_{n-1}\right) \geq \nu\left(m R^{b_{n}}\right)-\nu\left(R^{b_{n+1}}\right) \geq e b_{n}-b_{n+1} \geq e b-b=(e-1) b .
$$


Therefore, it follows from Lemma (2.5) that $I_{n-1}=m^{2} R^{b_{n-1}}$ for $n=i, i+1$, $i+2$. From the exact sequences

$$
0 \rightarrow \operatorname{Ker} d_{n} \rightarrow m R^{b_{n}} \stackrel{d_{n}}{\longrightarrow} I_{n-1} \longrightarrow 0
$$

we now get $l\left(\operatorname{Ker} d_{n}\right)=l\left(m R^{b_{n}}\right)-l\left(I_{n-1}\right)=2 e b-e b=e b=l\left(I_{n}\right)$ for the same values of $n$. However, since $I_{n} \subseteq \operatorname{Im} d_{n+1}=\operatorname{Ker} d_{n}$, this implies $I_{n}=\operatorname{Ker} d_{n}$ for $n=i, i+1$.

Thus, we have the exact sequence

$$
0 \longrightarrow I_{i+1} \longrightarrow R^{b_{i+1}} \stackrel{d_{i+1}}{\longrightarrow} I_{i} \longrightarrow 0
$$

which gives $(2 e+1) b=l\left(R^{b_{i+1}}\right)=l\left(I_{i+1}\right)+l\left(I_{i}\right)=2 e b$, a contradiction.

Proof of (1.1). Replacing $R$ by $R^{\prime}=R[X]_{m R[X]}$ and $M$ by $M^{\prime}=M \otimes_{R} R^{\prime}$ if necessary, we may assume $k$ is infinite. Then there exists (by [8, Theorems 14.13 and 14.14]) an $R$-regular sequence $a_{1}, \ldots, a_{d}(d=\operatorname{dim} R)$ such that the multiplicity of $R$ is equal to the length of $\bar{R}=R /\left(a_{1}, \ldots, a_{d}\right)$, and $a_{1}, \ldots, a_{d}$ form part of a minimal system of generators of $m$. Then $\operatorname{Tor}_{i}^{R}(M, \bar{R})=0$ for every $i>d$, hence the complex $\mathbf{F}_{>d} \otimes_{R} \bar{R}$ is exact. Thus $\mathbf{F}_{\geq d} \otimes_{R} \bar{R}$ is the minimal free resolution of $\operatorname{Syz}_{d}^{R}(M) \otimes_{R} \bar{R}$ over $\bar{R}$. Therefore $b_{n+d}^{R}(M)=b_{n}^{\bar{R}}\left(\operatorname{Syz}_{d}^{R}(M) \otimes_{R} \bar{R}\right)$ for $n \geq 0$. Thus replacing $R$ by $\bar{R}$ and $M$ by $\mathrm{Syz}_{d}^{R}(M) \otimes_{R} \bar{R}$, we have to establish the following claim:

Let $R$ be artinian with $e \geq 4$ and $l \leq 7$, or let $R$ be artinian Gorenstein with $e \geq 5$ and $l \leq 11$. If $M$ is a nonfree $R$-module, then the sequence $\left\{b_{n}^{R}(M)\right\}_{n \geq \nu(M)+1}$ is strictly increasing.

In the first case the claim follows directly from (2.3.ii). In the Gorenstein case (2.3.ii) applies when $h \geq 4$ or when $h \leq 2$. Assuming the claim fails for the remaining value $h=3$, we obtain from our assumptions and (2.6) that $12 \leq 2(e+1)=l \leq 11$, a contradiction.

\section{THE EXAMPLES}

We denote by $\operatorname{gr}_{m} R$ the associated graded ring $\bigoplus_{i \geq 0} m^{i} / m^{i+1}$ and say $R$ is graded if the rings $R$ and $\mathrm{gr}_{m} R$ are isomorphic; note that this implies $R$ is artinian. If $S=\bigoplus_{i} S_{i}$ is a graded ring and $M=\bigoplus_{i} M_{i}, N=\bigoplus_{i} N_{i}$ are graded $S$-modules, a homomorphism $\varphi: M \rightarrow N$ is an $S$-linear map, such that for all $i: \varphi\left(M_{i}\right) \subseteq N_{i}$. As usual, we write $M(a)$ to denote the graded $S$-module with $M(a)_{i}=M_{i+a}$.

(3.1) Proposition. Let $k$ be a field and let $\alpha$ be a nonzero element of $k$. Denote by $o(\alpha)$ the order of $\alpha$ in the group of units of $k$. Set $R=R_{\alpha}=$ $k\left[X_{1}, \ldots, X_{5}\right] / I_{\alpha}$, where the $X_{i}$ 's are indeterminates and $I=I_{\alpha}$ is the ideal 
generated by the following quadratic forms:

$$
\begin{aligned}
& \alpha X_{1} X_{3}+X_{2} X_{3}, \quad X_{1} X_{4}+X_{2} X_{4}, \quad X_{3}^{2}-X_{2} X_{5}+\alpha X_{1} X_{5}, \\
& X_{4}^{2}-X_{2} X_{5}+X_{1} X_{5}, \quad X_{1}^{2}, \quad X_{2}^{2}, \quad X_{3} X_{4}, \quad X_{3} X_{5}, \quad X_{4} X_{5}, \quad X_{5}^{2} .
\end{aligned}
$$

Denote by $x_{i}$ the image of $X_{i}$ in $R$. Then: $t^{3}$.

(i) $(R, m, k)$ is a graded local Gorenstein ring with $\mathrm{Hilb}_{R}(t)=1+5 t+5 t^{2}+$

(ii) The sequence of homomorphisms of graded $R$-modules

$$
\mathbf{G}=\mathbf{G}(\alpha): \cdots \leftarrow R^{2}(-n+1) \stackrel{d_{n}}{\longleftarrow} R^{2}(-n) \stackrel{d_{n+1}}{\longleftarrow} R^{2}(-n-1) \leftarrow \cdots,
$$

where $n \in \mathbf{Z}$,

$$
d_{n}=\left(\begin{array}{cc}
x_{1} & \alpha^{n} x_{3}+x_{4} \\
0 & x_{2}
\end{array}\right)
$$

is an exact complex (the ith column of the matrix of $d_{n}$ is the image of the ith vector of the standard basis).

(iii) Set $M=M_{\alpha}=\operatorname{Im} d_{0}$. If $o(\alpha)$ divides $t-s$, then

$$
\operatorname{Syz}_{s}^{R}(M) \cong \operatorname{Syz}_{t}^{R}(M)(t-s)
$$

as graded modules. Conversely, if $\operatorname{Syz}_{s}^{R}(M) \cong \operatorname{Syz}_{t}^{R}(M)$ as (not necessarily graded) $R$-modules, then $t-s \equiv 0 \bmod (o(\alpha))$.

The following examples are immediate consequences:

(3.2) Example. if $o(\alpha)=\infty$, then $M$ is not eventually periodic over $R$.

(3.3) Example. If $o(\alpha)=q$, then $M$ is periodic of minimal period $q$ over $R$.

Proof of (3.1). (i) Obviously, $R$ is a local ring graded by setting $\operatorname{deg} x_{i}=1$. Choose in $R_{1}$ the basis $\left\{x_{i} \mid 1 \leq i \leq 5\right\}$. Clearly $y_{5}=x_{1} x_{2}, y_{3}=x_{1} x_{3}$, $y_{4}=x_{1} x_{4}, y_{2}=x_{1} x_{5}, y_{1}=x_{2} x_{5}$ form a basis of $R_{2}$. Set $z=x_{1} x_{2} x_{5}$. A direct computation shows that $x_{i} y_{j}=\delta_{i j} z$ for $1 \leq i, j \leq 5$, and $m^{4}=0$.

In order to establish (i) we have only to prove that $x_{1} x_{2} x_{5} \neq 0$. Denote by $g_{1}, g_{2}, \ldots, g_{10}$ the generators of $I$, in their order of appearance in the statement of the proposition. If $x_{1} x_{2} x_{5}=0$, then $X_{1} X_{2} X_{5}=\sum_{i=1}^{10}\left(\sum_{j=1}^{5} \beta_{i j} X_{j}\right) g_{i}$ for some $\beta_{i j} \in k$. The following relations are provided by equating the coefficients of $X_{1} X_{2} X_{5}, X_{1} X_{3}^{2}, X_{2} X_{3}^{2}, X_{1} X_{4}^{2}$, and $X_{2} X_{4}^{2}$, respectively:

$$
\begin{gathered}
A=-\beta_{31}+\alpha \beta_{32}-\beta_{41}+\beta_{42}=1, \\
B=\beta_{31}+\alpha \beta_{13}=0, \quad C=\beta_{32}+\beta_{13}=0, \\
D=\beta_{24}+\beta_{41}=0, \quad E=\beta_{24}+\beta_{42}=0 .
\end{gathered}
$$

Now $0=E-D+\alpha C-B=-\beta_{31}+\alpha \beta_{32}-\beta_{41}+\beta_{42}=A=1$ gives the desired contradiction. 
(ii) It is immediate from the defining equations of $R$ that $d_{n} d_{n+1}=0$. Denote by $d_{n, i}$ the restriction of $d_{n}$ on $R^{2}(-n)_{i}$. The images by $d_{n, n+1}$ of $\left(x_{2}, 0\right),\left(x_{3}, 0\right),\left(x_{4}, 0\right),\left(x_{5}, 0\right),\left(0, x_{1}\right),\left(0, x_{3}\right),\left(0, x_{4}\right)$, and $\left(0, x_{5}\right)$ are linearly independent in $R^{2}(-n+1)_{n+1}$. Also $d_{n, n+2}\left(x_{2} x_{5}, 0\right)=(z, 0)$ and $d_{n, n+2}\left(0, x_{1} x_{5}\right)=(0, z)$. Thus,

$$
\operatorname{rank}_{k} d_{n} \geq \sum_{i=n}^{n+2} \operatorname{rank}_{k} d_{n, i} \geq 2+8+2=12 .
$$

On the other hand,

$$
\begin{aligned}
\operatorname{rank}_{k} d_{n} & =\operatorname{dim}_{k}\left(R^{2}(-n+1)\right)-\operatorname{dim}_{k}\left(\operatorname{Ker} d_{n-1}\right) \\
& \leq \operatorname{dim}_{k}\left(R^{2}(-n+1)\right)-\operatorname{rank}_{k} d_{n} \leq 24-12=12 .
\end{aligned}
$$

Thus, for any $n, \operatorname{dim}_{k}\left(\operatorname{Ker} d_{n}\right)=\operatorname{dim}_{k}\left(\operatorname{Im} d_{n}\right)=12$, which proves exactness.

(iii) Since the first assertion is obvious, assume $\operatorname{Syz}_{s}^{R}(M) \cong \operatorname{Syz}_{t}^{R}(M)$ as $R$ modules with, say, $s<t$. This lifts to an isomorphism of degree $s-t$ of complexes $u: \mathbf{G}_{\geq t} \rightarrow \mathbf{G}_{\geq s}$, where $G_{\geq r}$ denotes the complex $\cdots \leftarrow 0 \leftarrow 0 \leftarrow$ $G_{r} \stackrel{d_{r+1}}{\longleftarrow} G_{r+1} \leftarrow \cdots$. Let $u_{t}$ and $u_{t+1}$ be given in the standard basis by matrices:

$$
\left(\begin{array}{ll}
a & b \\
c & d
\end{array}\right) \text { and }\left(\begin{array}{ll}
a^{\prime} & b^{\prime} \\
c^{\prime} & d^{\prime}
\end{array}\right)
$$

respectively. Then by comparing the $(1,2)$ entry in the matrix equality $d_{s+1} u_{t+1}$ $=(-1)^{t-s} u_{t} d_{t+1}$, one has

$$
b^{\prime} x_{1}+\alpha^{s+1} d^{\prime} x_{3}+d^{\prime} x_{4}=(-1)^{t-s}\left(\alpha^{t+1} a x_{3}+a x_{4}+b x_{2}\right) .
$$

Let $\bar{v}$ be the image of $v \in R$ in $k=R / m$. Since $I$ is generated by quadratic forms,

$$
\bar{b}^{\prime} x_{1}+\alpha^{s+1} \bar{d}^{\prime} x_{3}+\bar{d}^{\prime} x_{4}=(-1)^{t-s}\left(\alpha^{t+1} \bar{a} x_{3}+\bar{a} x_{4}+\bar{b} x_{2}\right)
$$

holds. Hence $\bar{b}^{\prime}=0, \bar{b}=0, \alpha^{s+1} \bar{d}^{\prime}=(-1)^{t-s} \alpha^{t+1} \bar{a}$, and $\bar{d}^{\prime}=(-1)^{t-s} \bar{a}$. If we assume that $t-s \not \equiv 0 \bmod (o(\alpha))$, then $\bar{d}^{\prime}=\bar{a}=0$, so $u_{t}$ and $u_{t+1}$ are not isomorphisms, contradicting our assumption.

(3.4) Proposition. Let $k, \alpha, R_{\alpha}, M_{\alpha}, o(\alpha)$, and $\mathbf{G}(\alpha)$ be as in (3.1). Then:

(i) $\left(P=P_{\alpha}=R_{\alpha} /\left(x_{5}\right), m^{\prime}, k\right)$ is a local graded artinian ring with $m^{\prime 3}=0$.

(ii) The complex $\mathbf{H}(\alpha)=\mathbf{G}(\alpha) \otimes_{R_{\alpha}} P_{\alpha}$ is exact.

(iii) Let $L=L_{\alpha}=M_{\alpha} \otimes_{R_{\alpha}} P_{\alpha}$. Then $\operatorname{Syz}_{s}^{P}\left(L_{\alpha}\right) \cong \operatorname{Syz}_{t}^{P}\left(L_{\alpha}\right)$ if and only if $t-s \equiv 0 \bmod (o(\alpha))$.

The proof of (3.4) is omitted because it is similar to that of (3.1). 
In particular, we have the following examples:

(3.5) Example. If $o(\alpha)=\infty$, then $L$ is not eventually periodic over $P$.

(3.6) Example. If $o(\alpha)=q$, then $L$ is periodic of minimal period $q$ over $P$.

(3.7) Remark. From (1.0.ii) it follows that the rings in our examples have the minimal possible embedding dimension. It is easily seen, by taking tensor products over $k$ of $P$ and $R$ with appropriate $k$-algebras, that counterexamples to Eisenbud's conjecture exist over rings $P^{\prime}$, respectively Gorenstein rings $R^{\prime}$, with arbitrary values of the embedding dimension and depth, subject only to the conditions edim $P^{\prime}-\operatorname{depth} P^{\prime} \geq 4$ and, respectively, edim $R^{\prime}-\operatorname{depth} R^{\prime} \geq 5$.

It should be noted that in the counterexamples constructed above, the rings are graded $k$-algebras and have minimal length (by (1.2)) and minimal nilpotency degree of the maximal ideal (by (2.4)). The corresponding modules are graded, have linear resolutions, and are infinite syzygies (which is a strong necessary condition for periodicity). They have constant Betti numbers equal to 2 , which is the minimal possible value of $\lim \sup b_{n}$, as seen from the following:

(3.8) Proposition. If $M$ is an $R$-module such that $b_{n}^{R}(M)=1$ for $n \gg 0$, then $M$ is eventually periodic of period 2 .

Proof. By assumption, for $n \gg 0$, the differential of the minimal resolution of $M$ is $R \stackrel{x_{n}}{\longrightarrow} R$, for some $x_{n} \in R$. Since $x_{i-1} x_{i}=0$, one has $x_{i-1} \in$ $\left(0: x_{i}\right)=x_{i+1} R$ for sufficiently large $i$. Thus we have $x_{i-1} R \subseteq x_{i+1} R$. As $R$ is noetherian, the conclusion follows.

(3.9) Remark. In the examples, $m^{2} M=0$, and this certainly is "minimal" in the following sense. If $m M=0$, i.e., $M$ is a direct sum of copies of the residue field, and $M$ has bounded Betti numbers, then it is well known that $R$ is a hypersurface ring; hence by $[5,(6.1)], M$ is eventually periodic of period 2 . In particular, for any counterexample to Eisenbud's conjecture one has length $M>1$, but we do not know what the minimal possible length is.

(3.10) Remark. In [1] a generalization of the notion of projective dimension of a module, called virtual projective dimension and noted $\operatorname{vpd}_{R} M$, is introduced. By [1, (4.4)], a module which has finite virtual projective dimension and bounded Betti numbers is eventually periodic of period 2. Thus, for $\alpha \neq \pm 1$, one has $\operatorname{vpd}_{R_{\alpha}} M_{\alpha}=\infty$ and $\operatorname{vpd}_{P_{\alpha}} L_{\alpha}=\infty$. In fact, for $\alpha \neq \pm 1$ the ring $P_{\alpha}$ has no embedded deformation: this can be checked by the same argument as used in [3].

However, for $\alpha= \pm 1$ (3.4) does not provide modules with bounded Betti numbers and infinite virtual projective dimension. To show this, we exhibit an embedded codimension 1 deformation $Q_{\alpha}$ of $P_{\alpha}$ such that $\mathrm{pd}_{Q_{\alpha}} L_{\alpha}=1$ : by [1] this means $\operatorname{vpd}_{P_{\alpha}} L_{\alpha}=1$. Set $Q_{\alpha}^{\prime}=k\left[X_{1}, X_{2}, X_{3}, X_{4}\right] / T$ where the $X_{i}$ 's are indeterminates and $T$ is the ideal generated by the following quadratic 
forms:

$$
\alpha X_{1} X_{3}+X_{2} X_{3}, \quad X_{1} X_{4}+X_{2} X_{4}, \quad X_{3}^{2}, \quad X_{4}^{2}, \quad X_{3} X_{4}, \quad X_{1}^{2}-X_{2}^{2} .
$$

Denote by $x_{i}$ the image of $X_{i}$ in $Q_{\alpha}^{\prime}$ and by $Q_{\alpha}$ the localization at $\left(x_{1}, x_{2}, x_{3}\right.$, $\left.x_{4}\right)$ of $Q_{\alpha}^{\prime}$. Then $x_{1}^{2}$ is a nonzero divisor of $Q_{\alpha}$, and $P_{\alpha}=Q_{\alpha} /\left(x_{1}^{2}\right)$, hence $Q_{\alpha}$ is an embedded deformation of $P_{\alpha}$. The minimal free resolution of $L_{\alpha}$ over $Q_{\alpha}$ is then

$$
Q_{\alpha}^{2} \stackrel{\left(\begin{array}{cc}
x_{1} & \alpha x_{3}+x_{4} \\
0 & x_{2}
\end{array}\right)}{\longleftarrow} Q_{\alpha}^{2} \leftarrow 0 .
$$

(3.11) Remark. Note that for $\alpha=-1$ (respectively $\alpha=1$ ) the module $L_{\alpha}$ is periodic of minimal period 2 (respectively 1 ). Thus, the questions may arise whether a module of minimal period 2 (respectively 1 ) necessarily has finite virtual projective dimension. Once more, the answers are negative. For period 2 the relevant example is constructed in [3]. For period 1, take in (3.4) $\alpha \neq \pm 1$ and set $N_{\alpha}=P_{\alpha} /\left(x_{1}\right)$. The complex

$$
P_{\alpha} \stackrel{x_{1}}{\longleftarrow} P_{\alpha} \stackrel{x_{1}}{\longleftarrow} P_{\alpha} \longleftarrow \cdots
$$

is then the minimal $P_{\alpha}$-free resolution of $N_{\alpha}$.

\section{ARTINIAN RINGS WITH SMALl RESIDUE FIELD}

The examples (3.2) and (3.5) of nonperiodic modules with bounded Betti numbers require that $k$ contain an element of infinite multiplicative order, i.e., that $k$ be not algebraic over a finite field. That this condition is essential is shown by (4.2) below.

In (3.3) and (3.6) the assumption that $k$ contains a $q$ th root of unity can be avoided at the expense of increasing the embedding dimension. This is shown in (4.3).

(4.1) Proposition. Let $R$ be artinian, such that $k$ is an algebraic extension of the prime field $\mathbf{F}_{p}(p>0)$. For any finitely generated $R$-module $M$ there are a finite artinian ring $R^{\prime}$ and an $R^{\prime}$-module $M^{\prime}$ such that $R$ is a faithfully flat $R^{\prime}$-module, and $M=M^{\prime} \otimes_{R^{\prime}} R$.

Remark. In the case when $R$ contains a field, the proposition is an immediate consequence of the proof of $[6,(2.2)]$.

Proof. From Cohen's structure theorem [4, $\S 2$, Théorème 3] it follows that $R \cong$ $S / I$, where $S=V\left[X_{1}, \ldots, X_{n}\right], V$ is a complete discrete valuation ring with maximal ideal $p V, V / p V \cong k$, and $I=\left(f_{1}, \ldots, f_{r}\right) S \supseteq\left(p, X_{1}, \ldots, X_{n}\right)^{u}$ for some $u \geq 1$. Since $k$ is perfect there is [4, $\S 2$, Proposition 7] a multiplicative system $A=\left\{a_{\lambda}, \lambda \in \Lambda\right\} \cong k^{*}$ such that every $v \in V$ is expressed uniquely 
in the form $v=\sum_{h=0}^{\infty} a_{v, h} p^{h}, a_{v, h} \in A \cup\{0\}$. For $v \in V$ set $A_{v}=\left\{a_{v, h} \in\right.$ $A \mid h<u\}$ and for $f \in S$ let $A_{f}$ denote the union of all $A_{v}$, taken over the coefficients of $f$. Note that $\left|A_{f}\right|<\infty$. Let

$$
0 \leftarrow M \leftarrow S^{t} \stackrel{\left(g_{i j}\right)}{\longleftarrow} S^{d}
$$

be a presentation of $M$ and let $B$ be the multiplicative subgroup of $A$ generated by the finite set $\left(\bigcup_{1 \leq i \leq r} A_{f_{i}}\right) \cup\left(\bigcup_{1 \leq i \leq t, 1 \leq j \leq d} A_{g_{i j}}\right)$. Since $k$ is an algebraic extension of $\mathbf{F}_{p}$, every element of $k^{*}$ is torsion, hence so is every element of $A$, hence $B$ is finite. It follows that there exists a $b \in B$ such that $B=\langle b\rangle$. With the $p$-adic numbers $\mathbf{Z}_{p}$, canonically embedded in $V$, set $V^{\prime}=\mathbf{Z}_{p}[b]$. Since $V^{\prime}$ is integral over $\mathbf{Z}_{p}$ and $V^{\prime} / p V^{\prime}$ is the field $\mathbf{F}_{p}(\bar{b})$ (where $\bar{b}$ is the image of $b$ in $k=V / p V)$, one sees that $V^{\prime}$ is a complete discrete valuation ring with maximal ideal $p V^{\prime}$. The domain $V$ is a torsion-free $V^{\prime}$-module and the inclusion $V^{\prime} \rightarrow V$ is flat. Note now that $f_{h}, 1 \leq h \leq r$, and $g_{i j}, 1 \leq i \leq d$, $1 \leq j \leq t$, are contained in $S^{\prime}=V^{\prime}\left[X_{1}, \ldots, X_{n}\right]$. Setting $R^{\prime}=S^{\prime} /\left(f_{1}, \ldots, f_{r}\right)$ and

$$
M^{\prime}=\operatorname{Coker}\left(S^{\prime d} \stackrel{\left(g_{i j}\right)}{\longrightarrow} S^{\prime t}\right)
$$

we have $M=M^{\prime} \otimes_{R^{\prime}} R$ and $R=R^{\prime} \otimes_{V^{\prime}} V$. Obviously, $R^{\prime}$ is a finite local artinian ring and $R^{\prime} \rightarrow R$ is flat.

(4.2) Corollary. Let $R$ be as in (4.1) and let $M$ be an $R$-module with $\operatorname{cx}_{R} M=$ 1. Then $M$ is eventually periodic.

Proof. With the $R^{\prime}$ and $M^{\prime}$ given by (4.1), let $\mathbf{F}^{\prime}$ be a minimal free resolution of $M^{\prime}$ over $R^{\prime}$. Since $R^{\prime}$ is $R$-flat, $\mathbf{F}=\mathbf{F}^{\prime} \otimes_{R} R^{\prime}$ is a minimal free resolution of $M$ over $R$, so it is enough to show that $M^{\prime}$ is eventually periodic. But $b_{n}^{R^{\prime}}\left(M^{\prime}\right)=b_{n}^{R}(M)$ are bounded and $R^{\prime}$ is a finite ring, hence there is only a finite number of possibilities for the matrices $d_{n}^{\prime}$. Thus $M^{\prime}$ is eventually periodic.

(4.3) Example. Let $k$ be an arbitrary field. Set

$$
S_{n}=k\left[X_{1}, X_{2}, X_{3}, Y_{1}, \ldots, Y_{n}\right] / I_{n},
$$

where the $X_{i}$ 's and $Y_{i}$ 's are indeterminates and $I_{n}$ is the ideal generated by the following quadratic forms:

$$
\begin{gathered}
X_{1} Y_{1}+X_{2} Y_{n} ; X_{1} Y_{i}+X_{2} Y_{i-1} \quad \text { for } 2 \leq i \leq n \\
X_{2} X_{3}-Y_{1}^{2} ; X_{2} X_{3}-Y_{i} Y_{n+2-i} \quad \text { for } 2 \leq i \leq 1+n / 2 \\
X_{1} X_{3}+Y_{i} Y_{n+1-i} \quad \text { for } 1 \leq i \leq(n+1) / 2 \\
X_{1}^{2}, X_{2}^{2}, X_{3}^{2} ; Y_{i} X_{3} \quad \text { for } 1 \leq i \leq n ; \\
Y_{i} Y_{j} \quad \text { for } 1 \leq i \leq j \leq n, i+j \neq n+1, n+2 \text { and }(i, j) \neq(1,1) .
\end{gathered}
$$


Denote by $x_{i}$ and $y_{j}$, respectively, the images of $X_{i}$ and $Y_{j}$ in $S_{n}$; then:

(i) $S=\left(S_{n}, m_{n}, k\right)$ is a local graded Gorenstein ring with $\operatorname{Hilb}_{S}(t)=1+$ $(n+3) t+(n+3) t^{2}+t^{3}$.

(ii) The sequence of homomorphisms of graded $R$-modules

$$
\begin{gathered}
\mathbf{C}_{n}: \cdots \leftarrow S^{2}(-i+1) \stackrel{d_{i}}{\leftarrow} S^{2}(-i) \leftarrow \cdots, \text { where } i \in \mathbf{Z}, \\
d_{i}=\left(\begin{array}{cc}
x_{1} & y_{i} \\
0 & x_{2}
\end{array}\right)
\end{gathered}
$$

for $y_{i}=y_{j}$ if $i \equiv j \bmod (n)$, is an exact complex.

(iii) Let $N=N_{n}=\operatorname{Im} d_{0}$; then $N$ is a periodic module over $S$ of minimal period $n$.

Setting $S^{\prime}=S /\left(x_{3}\right)$, we obtain as in $\S 3$ an example over a non-Gorenstein ring.

\section{REFERENCES}

1. L. L. Avramov, Modules of finite virtual projective dimension, Invent. Math. 96 (1989), $71-101$

2. __ Homological asymptotics of modules over local rings, Commutative Algebra (M. Hochster, C. Huneke, J. Sally, Eds.), MSRI Publications, vol. 15, Springer-Verlag, 1989, pp. 33-62.

3. L. L. Avramov, V. N. Gasharov, and I. V. Peeva, A periodic module of infinite virtual projective dimension, J. Pure Appl. Algebra 62 (1989), 1-5.

4. N. Bourbaki, Algèbre commutative, Chap. 9, Masson, Paris, 1983.

5. D. Eisenbud, Homological algebra on a complete intersection with an application to group representations, Trans. Amer. Math. Soc. 260 (1980), 35-64.

6. C. Jacobsson and V. Stoltenberg-Hansen, Poincaré-Betti series are primitive-recursive, J. London Math. Soc. (2) 31 (1985), 1-9.

7. J. Lescot, Asymptotic properties of Betti numbers of modules over certain rings, J. Pure Appl. Algebra 38 (1985), 287-298.

8. H. Matsumura, Commutative ring theory, Cambridge Univ. Press, 1986.

Section of Algebra, Institute of Mathematics, University of Sofia, 1113 Sofia, BULGARIA 